\title{
Chern-Simons-like action induced radiatively in General Relativity
}

\author{
T. Mariz, J. R. Nascimento, E. Passos and R. F. Ribeird* \\ Departamento de Física, Universidade Federal da Paraíba, \\ Caixa Postal 5008, 58051-970 João Pessoa, Paraíba, Brazil
}

(Dated: November 6, 2018)

\begin{abstract}
The Chern-Simons-like gravitational action is evaluated explicitly in four dimensional space-time by radiative corrections at one-loop level. The calculation is performed in fermionic sector where the Dirac fermions interact with the background gravitational field, including the parity-violating term $\bar{\psi} \not b \gamma_{5} \psi$. The investigation takes into account the weak field approximation and dimensional regularization scheme.

PACS numbers:
\end{abstract}

*Electronic address: tiago, jroberto, passos, rfreire@fisica.ufpb.br 


\section{INTRODUCTION}

Theory of the electromagnetism was crucial to question Galilei invariance, to give rise to Lorentz symmetry. Nowadays, in string theory one may find a way to question Lorentz invariance, since there are interactions that support spontaneous breaking of Lorentz symmetry [1], one of those interactions being described by the Chern-Simons-like action.

The Chern-Simons term was first introduced in three-dimensional gauge field and gravitational field theories by Deser, Jackiw and Templeton [2]. In the gauge theories interesting phenomena are exhibited such as exotic statistics, fractional spin and massive gauge field. These phenomena are of topological nature and they can be produced when we add ChernSimons term to Lagrangian which describe the system under consideration. Posteriorly, was observed that if one adds the Chern-Simons-like term in four dimensional space-time to Maxwell's theory both Lorentz and CPT symmetries are violated. The model predicts the rotation of the plane of polarization of radiation from distance galaxies, an effect which is not observed yet [3]. In recent papers [4, 5], it has been studied the modification of general relativity when one adds the Chern-Simons-like gravitational term. The authors have observed that in this modified theory the Schwarzschild metric is solution, gravitational waves possess two polarizations which travel with the velocity of light and polarized waves are suppressed or enhanced.

The induction by radiative correction of the Chern-Simons term has been analyzed in the last twenty years. Redlich [6] in a seminal paper studied the subject in the context of the quantum electrodynamics in three-dimensional of space-time. Following this paper other models in quantum field theory were investigated [7, 8, 9]. Extension to higher odd-dimension was done and also in the case of a gravitational background field [10, 11].

Colladay and Kostelecky [12] analyzed the question whether Chern-Simons-like term is generated by radiative corrections when Lorentz and CPT violating term $\bar{\psi} b_{\mu} \gamma^{\mu} \gamma_{5} \psi$ is added to the conventional Lagrangian of quantum electrodynamics in four-dimensional space-time. They have observed that such term is dependent on regularization scheme. Coleman and Glashow[13] argued that such term must unambiguously vanish to first order in $b_{\mu}$ for any gauge invariant CPT odd interaction. They considered that the axial current $j_{\mu}^{5}$ should keep gauge invariant in the quantum theory at any momentum or at any space-time point. Since $\left\langle j_{\mu}^{5}\right\rangle=\delta L(x) / \delta b_{\mu}$, this condition is equivalent to the requirement that the Lagrangian den- 
sity corresponding to the quantum effective action should be gauge invariant. Thus, based on this requirement the Chern-Simons-like term is not generated since its Lagrangian density is explicitly not gauge invariant. Jackiw and Kostelecký[14] shown that Chern-Simons-like term is induced. They thought that since $j_{\mu}^{5}$ only couples with a constant 4 -vector $b_{\mu}$, it is true to require only that $j_{\mu}^{5}$ with zero-momentum is gauge invariant at quantum level. Since $\left\langle\int d^{4} x j_{\mu}^{5}\right\rangle=\delta S / \delta b_{\mu}$, this condition is equivalent to the requirement that the quantum effective action should be gauge invariant. This controversy on a possible Chern-Simons-like term generated through radiative corrections was carefully investigated by many authors 15, 16, 17, 18, 19, 20, 21, 22, 23, 24, 25, 26]. This phenomena was analyzed in quantum electrodynamics as a part of the standard model. Our purpose in this paper is to derive the Chern-Simons-like gravitational action induced by Dirac fermions coupled to a background gravitational field. The result show that the Chern-Simons-like term generated by radiative fermion loops, under the assumption of the weak field approximation and dimensional regularization scheme.

\section{EVALUATING THE CHERN-SIMONS-LIKE GRAVITATIONAL ACTION}

The action that we are interested is given by

$$
S=\int \mathrm{d}^{4} x\left(\frac{1}{2} i e e_{a}^{\mu} \bar{\psi} \gamma^{a} \stackrel{\leftrightarrow}{D}_{\mu} \psi-e e_{a}^{\mu} \bar{\psi} b_{\mu} \gamma^{a} \gamma_{5} \psi\right)
$$

where we have included the parity-violating term. Here, $e^{\mu}{ }_{a}$ is the tetrad (vierbein), $e \equiv$ $\operatorname{det} e^{\mu}{ }_{a}$ and $b_{\mu}$ is a constant 4 -vector. The covariant derivative is given by

$$
D_{\mu} \psi=\partial_{\mu} \psi+\frac{1}{2} w_{\mu c d} \sigma^{c d} \psi
$$

where $w_{\mu}{ }^{c d}$ is the spin connection and $\sigma^{c d}=\frac{1}{4}\left[\gamma^{c}, \gamma^{d}\right]$, whereas the covariant derivative on a Dirac-conjugate field $\bar{\psi}$ is

$$
D_{\mu} \bar{\psi}=\partial_{\mu} \bar{\psi}-\frac{1}{2} w_{\mu c d} \bar{\psi} \sigma^{c d}
$$

Using the expressions above we can rewrite the Eq. (II) as follow

$$
S=\int \mathrm{d}^{4} x\left(\frac{1}{2} i e e_{a}^{\mu} \bar{\psi} \gamma^{a} \stackrel{\leftrightarrow}{\partial}_{\mu} \psi+\frac{1}{4} i e e_{a}^{\mu} \bar{\psi} w_{\mu c d} \Gamma^{a c d} \psi-e e_{a}^{\mu} \bar{\psi}_{\mu} \gamma^{a} \gamma_{5} \psi\right)
$$

where $\Gamma^{a c d}=\frac{1}{6}\left(\gamma^{a} \gamma^{c} \gamma^{d} \pm\right.$ permutations $)$, i.e. the antisymmetrized product of three $\gamma$ matrices. 
In the weak field approximation we consider $g_{\mu \nu}=\eta_{\mu \nu}+h_{\mu \nu}\left(g^{\mu \nu}=\eta^{\mu \nu}-h^{\mu \nu}\right)$, which induces an expansion for the vierbein $e_{\mu a}=\eta_{\mu a}+\frac{1}{2} h_{\mu a}\left(e_{a}^{\mu}=\eta_{a}^{\mu}-\frac{1}{2} h_{a}^{\mu}\right)$. Then, the linearized Chern-Simons-like action takes the form [4]

$$
S_{\text {linear }}=\frac{1}{4} \int d^{4} x h^{\mu \nu} v^{\lambda} \epsilon_{\alpha \mu \lambda \rho} \partial^{\rho}\left(\partial_{\gamma} \partial^{\gamma} h_{\nu}^{\alpha}-\partial_{\nu} \partial_{\gamma} h^{\gamma \alpha}\right),
$$

The main purpose of the present work is to induce this action by radiative correction of fermionic matter field to obtain the relation between $v_{\lambda}$ and $b_{\mu}$. In order to perform this calculation we consider the fermionic model represented by the action

$$
e^{i \Gamma[h]}=\int \mathcal{D} \bar{\psi} \mathcal{D} \psi e^{i S[h, \bar{\psi}, \psi]},
$$

where the linearized effective action is given by

$$
S[h, \bar{\psi}, \psi]=\int \mathrm{d}^{4} x\left(\frac{1}{2} i \bar{\psi} \Gamma^{\mu} \stackrel{\leftrightarrow}{\partial}_{\mu} \psi+\bar{\psi} h_{\mu \nu} \Gamma^{\mu \nu} \psi-\bar{\psi} b_{\mu} \gamma^{\mu} \gamma_{5} \psi\right)
$$

with $\Gamma^{\mu}=\gamma^{\mu}-\frac{1}{2} h^{\mu \nu} \gamma_{\nu}$ and $\Gamma^{\mu \nu}=\frac{1}{2} b^{\mu} \gamma^{\nu} \gamma_{5}-\frac{i}{16}\left(\partial_{\rho} h_{\alpha \beta}\right) \eta^{\beta \nu} \Gamma^{\rho \mu \alpha}$. In this expression, we neglect the terms proportional to $h=\eta^{\mu \nu} h_{\mu \nu}$ because they do not contribute to generate the Chern-Simons-like action.

The Feynman rules that we obtain from Eq.(7) are:

Fermion propagator

$$
\longrightarrow \quad=S(p)=\frac{i}{\not p-m},
$$

Fermion propagator with $\not b$ insertion

$$
\rightarrow \quad=-i \not \gamma \gamma_{5}
$$

The three relevant interaction fermion-graviton vertices are

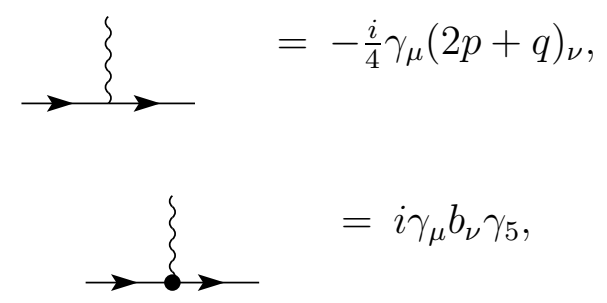

and

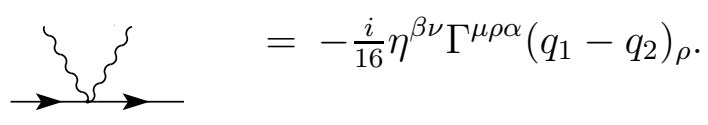



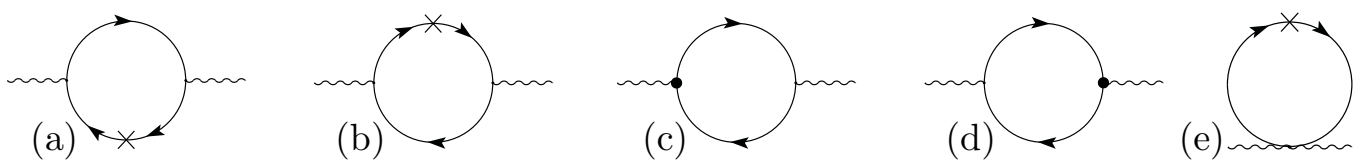

FIG. 1: One-loop contributions

The one-loop order correction to the effective action will be given by the graphs below.

The graphs (c), (d) and (e) do not contribute to generate Chern-Simons-like action. The only relevant graphs are (a) and (b) whose the Feynman integral are given by

$$
\Pi_{a}^{\mu \nu \alpha \beta}(q)=-\frac{i}{16} \operatorname{tr} \int \frac{\mathrm{d}^{4} p}{(2 \pi)^{4}}\left[\gamma^{\mu}(2 p+q)^{\nu} S(p) \gamma^{\alpha}(2 p+q)^{\beta} S(p+q) \not b \gamma_{5} S(p+q)\right]
$$

and

$$
\Pi_{b}^{\mu \nu \alpha \beta}(q)=-\frac{i}{16} \operatorname{tr} \int \frac{\mathrm{d}^{4} p}{(2 \pi)^{4}}\left[\gamma^{\mu}(2 p+q)^{\nu} S(p) \not b \gamma_{5} S(p) \gamma^{\alpha}(2 p+q)^{\beta} S(p+q)\right]
$$

It is straightforward to see that

$$
\Pi^{\mu \nu \alpha \beta}(q)=\Pi_{a}^{\mu \nu \alpha \beta}(q)=\Pi_{b}^{\alpha \nu \mu \beta}(-q)
$$

which appears of substituting the when loop momenta, $p \rightarrow p-q x$, and we using the cyclic properties of the trace of a product of $\gamma$-matrices. So, from now on we work only with Eq. (13) which takes the form

$$
\begin{aligned}
\Pi^{\mu \nu \alpha \beta}(q)= & -\frac{1}{8} \int_{0}^{1} \mathrm{~d} x x \int \frac{\mathrm{d}^{4} p}{(2 \pi)^{4}} \frac{(2 p+q(1-2 x))^{\nu}(2 p+q(1-2 x))^{\beta}}{\left[p^{2}-m^{2}+x(1-x) q^{2}\right]^{3}} \\
& \times \operatorname{tr}\left[\gamma^{\mu}(\not p-\not q x+m) \gamma^{\alpha}(\not p+\not q(1-x)+m) \not b \gamma_{5}(\not p+\not q(1-x)+m)\right] .
\end{aligned}
$$

where we have used Feynman parameter to combine the denominator in Eq. (16). First of all, to simplify the numerator of Eq. (16) we take into account that the trace of an odd product of $\gamma$ matrices times $\gamma_{5}$ is zero. Thus we have

$$
\begin{aligned}
\Pi^{\mu \nu \alpha \beta}(q)= & -\frac{1}{8} \int_{0}^{1} \mathrm{~d} x x \int \frac{\mathrm{d}^{4} p}{(2 \pi)^{4}} \frac{(2 p+q(1-2 x))^{\nu}(2 p+q(1-2 x))^{\beta}}{\left[p^{2}-m^{2}+x(1-x) q^{2}\right]^{3}} \\
& \times \operatorname{tr}\left[(\not p+\not q(1-x)) \gamma^{\mu}(\not p-\not 1 x) \gamma^{\alpha}(\not p+\not q(1-x)) \not b \gamma_{5}+m^{2}(\not p+\not q(1-x)) \gamma^{\mu} \gamma^{\alpha} \not b \gamma_{5}\right. \\
& \left.+m^{2} \gamma^{\mu}(\not p-\not q x) \gamma^{\alpha} \not b \gamma_{5}+m^{2} \gamma^{\mu} \gamma^{\alpha}(\not p+\not q(1-x)) \not \gamma \gamma_{5}\right] .
\end{aligned}
$$

Another algebraic properties of the $\gamma$-matrices are used to calculate the numerator of the Eq. (17). For instance, we can use that the trace of $\gamma_{5}$ times an even number of $\gamma$-matrices 
can be reduced: $\operatorname{Tr}\left[\gamma^{\mu} \gamma^{\nu} \gamma^{\alpha} \gamma^{\beta} \gamma_{5}\right]=4 i \epsilon^{\mu \nu \alpha \beta}$ and $\operatorname{Tr}\left[\gamma^{\mu} \gamma^{\nu} \gamma_{5}\right]=\operatorname{Tr}\left[\gamma_{5}\right]=0$. Also, we dropp all terms that are odd in $p$ to get

$$
\Pi^{\mu \nu \alpha \beta}(q)=-\frac{1}{8} \int_{0}^{1} d x x \int \frac{d^{4} p}{(2 \pi)^{4}} \frac{N^{\mu \nu \alpha \beta}\left(p^{0}, p^{2}, p^{4}\right)}{\left[p^{2}-m^{2}+x(1-x) q^{2}\right]^{3}} .
$$

When the numerator $N^{\mu \nu \alpha \beta}\left(p^{0}, p^{2}, p^{4}\right)$ has the form,

$$
\begin{aligned}
N^{\mu \nu \alpha \beta}\left(p^{0}, p^{2}, p^{4}\right) & =4 p^{\nu} p^{\beta}\left(T_{0}^{\alpha \mu}+T_{p p}^{\alpha \mu}\right)+2(1-2 x)\left(p^{\nu} q^{\beta}+p^{\beta} q^{\nu}\right)\left(T_{p}^{\alpha \mu}+T_{p p p}^{\alpha \mu}\right) \\
& +(1-2 x)^{2} q^{\nu} q^{\beta}\left(T_{0}^{\alpha \mu}+T_{p p}^{\alpha \mu}\right)
\end{aligned}
$$

with

$$
\begin{aligned}
T_{0}^{\alpha \mu}= & -4 i b_{\lambda} \epsilon^{\alpha \mu \lambda \theta} q_{\theta}\left[x(1-x)^{2} q^{2}+(2-x) m^{2}\right] \\
T_{p}^{\alpha \mu}= & -4 i b_{\lambda} \epsilon^{\alpha \mu \lambda \rho}\left[m^{2}+\left(1-x^{2}\right) q^{2}\right] p_{\rho} \\
& -8 i(1-x) b_{\lambda}\left[\epsilon^{\mu \lambda \rho \theta} q^{\alpha}-\epsilon^{\alpha \lambda \rho \theta} q^{\mu}-(1-x) \epsilon^{\alpha \mu \lambda \theta} q^{\rho}\right] q_{\theta} p_{\rho} \\
T_{p p}^{\alpha \mu}= & -4 i b_{\lambda}\left[2\left(\epsilon^{\mu \lambda \rho \theta} p_{\rho} p^{\alpha}-\epsilon^{\alpha \lambda \rho \theta} p_{\rho} p^{\mu}+x \epsilon^{\alpha \mu \lambda \rho} p_{\rho} p^{\theta}\right)-(2-x) \epsilon^{\alpha \mu \lambda \theta} p^{2}\right] q_{\theta} \\
T_{p p p}^{\alpha \mu}= & 4 i b_{\lambda} \epsilon^{\alpha \mu \lambda \rho} p^{2} p_{\rho}
\end{aligned}
$$

The integral (18) is badly divergent. By power counting we note that the kind of divergences are quadratic and logarithmic. Pehaps the mostconvenient method for regulating divergent integrals without impairing gauge invariance is the dimensional regularization scheme developed by 't Hooft and Veltman 27] in 1972. Thus, we change dimensions from 4 to $D$ and we change $d^{4} p /(2 \pi)^{4}$ to $\left(\mu^{2}\right)^{(2-D / 2)}\left[d^{D} p /(2 \pi)^{D}\right]$, where $\mu^{2}$ is an arbitrary parameter that identifies the mass scale. Thus Eq. (17) takes the form

$$
\Pi^{\mu \nu \alpha \beta}(q)=b_{\lambda} \epsilon^{\alpha \mu \lambda \rho} q_{\rho}\left[A q^{2} \eta^{\beta \nu}+B q^{\beta} q^{\nu}\right]
$$

where $A$ and $B$ are given by

$$
A=\frac{-1}{32 \pi^{2}} \int_{0}^{1} \mathrm{~d} x\left[(3-2 x) x^{2}(1-x) \Gamma(\epsilon / 2)-3 x^{2} \frac{M^{2}}{q^{2}} \Gamma(-1+\epsilon / 2)\right]\left(\frac{4 \pi \mu^{2}}{-M^{2}}\right)^{\epsilon / 2}
$$

and

$$
\begin{aligned}
B & =\frac{-2}{32 \pi^{2}} \int_{0}^{1} \mathrm{~d} x(1-2 x) x^{2}(1-x) \Gamma(\epsilon / 2)\left(\frac{4 \pi \mu^{2}}{-M^{2}}\right)^{\epsilon / 2} \\
& +\frac{1}{64 \pi^{2}} \int_{0}^{1} \mathrm{~d} x(1-2 x)^{2}\left[x(2-3 x)+(3-2 x) x^{2}(1-x) \frac{q^{2}}{M^{2}} \frac{\epsilon}{2}\right] \Gamma(\epsilon / 2)\left(\frac{4 \pi \mu^{2}}{-M^{2}}\right)^{\epsilon / 2}
\end{aligned}
$$


where $\epsilon=4-D$ and $M^{2}=m^{2}-x(1-x) q^{2}$. The next step concern expanding the gamma function that appear in $A$ and $B$ around $\epsilon \rightarrow 0$, thus we have

$$
\begin{aligned}
A & =\frac{1}{32 \pi^{2}} \int_{0}^{1} \mathrm{~d} x\left[3 x^{3}(1-x)+(5 x-3) x^{2}(1-x)\left(\frac{2}{\epsilon}+\ln \left(\frac{4 \pi \mu^{2}}{-M^{2}}\right)-\gamma\right)\right. \\
& \left.-3 x^{2}\left(\frac{2}{\epsilon}+\ln \left(\frac{4 \pi \mu^{2}}{-M^{2}}\right)-\gamma+1\right) \frac{m^{2}}{q^{2}}\right]
\end{aligned}
$$

and

$$
\begin{aligned}
B & =\frac{1}{64 \pi^{2}} \int_{0}^{1} \mathrm{~d} x\left\{(1-2 x)^{2}(3-2 x) \frac{x^{2}(1-x) q^{2}}{M^{2}}\right. \\
& \left.+[(2-3 x)(1-2 x)-4 x(1-x)] x(1-2 x)\left(\frac{2}{\epsilon}+\ln \left(\frac{4 \pi \mu^{2}}{-M^{2}}\right)-\gamma\right)\right\} .
\end{aligned}
$$

As one can see $\int_{0}^{1} d x\left[(5 x-3) x^{2}(1-x)\right]\left(\frac{2}{\epsilon}-\gamma\right)=0$ in $A$ and $\int_{0}^{1} d x[(2-3 x)(1-2 x)-4 x(1-$ $x)] x(1-2 x)\left(\frac{2}{\epsilon}-\gamma\right)=0$ in $B$, then $A$ and $B$ take the form

$$
\begin{aligned}
A & =\frac{1}{32 \pi^{2}} \int_{0}^{1} \mathrm{~d} x\left[3 x^{3}(1-x)+\frac{(1-2 x) x^{3}(1-x)^{2} q^{2}}{m^{2}-x(1-x) q^{2}}\right. \\
& \left.-3 x^{2}\left(\frac{2}{\epsilon}+\ln \left(\frac{4 \pi \mu^{2}}{-M^{2}}\right)-\gamma+1\right) \frac{m^{2}}{q^{2}}\right]
\end{aligned}
$$

and

$$
B=\frac{1}{32 \pi^{2}} \int_{0}^{1} \mathrm{~d} x \frac{(1-2 x)^{2} x^{2}(1-x) q^{2}}{m^{2}-x(1-x) q^{2}}
$$

Observe that we have performed an integration by parts on $x$ for $\log$ term in $A$ and $B$. Note that in $A$ the divergent part is present which will disappear when we consider the limit $m^{2} \rightarrow 0$. Now performing the $x$-integration, we have

$$
A \stackrel{m^{2} \rightarrow 0}{=}-B \stackrel{m^{2} \rightarrow 0}{=} \frac{1}{192 \pi^{2}} \text {. }
$$

We use these results into Eq. (24), to obtain the Chern-Simons-like term

$$
\Pi^{\mu \nu \alpha \beta}(q)=\frac{1}{192 \pi^{2}} b_{\lambda} \epsilon^{\alpha \mu \lambda \rho} q_{\rho}\left[q^{2} \eta^{\beta \nu}-q^{\beta} q^{\nu}\right]
$$

Finally, the Chern-Simons-like gravitational action induced by radiatively corrections is given by

$$
\Gamma_{\mathrm{cs}}[h]=\frac{1}{192 \pi^{2}} \int \mathrm{d}^{4} x b^{\lambda} h^{\mu \nu} \epsilon_{\alpha \mu \lambda \rho} \partial^{\rho}\left[\partial_{\gamma} \partial^{\gamma} h_{\nu}^{\alpha}-\partial_{\nu} \partial_{\gamma} h^{\gamma \alpha}\right],
$$

Comparing to Eq.(15) we obtain the relation between the parameters $v_{\lambda}$ and $b_{\mu}$ which is written as

$$
v_{\lambda}=\frac{1}{48 \pi^{2}} b_{\lambda}
$$




\section{CONCLUSIONS}

We summarize our work recalling that we have calculated the radiative corrections induced by Dirac fermions coupled to gravitational background field, including the nonstan-

dard contribution $\bar{\psi} \not \gamma_{5} \psi$ that violate parity symmetries. In this calculation we have used the weak field approximation and dimensional regularization scheme. The coefficient of the Chern-Simons-like gravitational action obtained in Eq. (34) is in agreement with the result obtained by Avarez-Gaumé and Witten within the context of gravitational anomalies [28].

\section{Acknowledgments}

We would like to thank D. Bazeia for useful discussions, CAPES, CNPq and PROCAD for partial support.

[1] V. A. Kostelecký and S. Samuel Phys. Rev. Lett. 63224 (1989).

[2] S. Deser, R. Jackiw, and S. Templeton, Ann. Phys. (N.Y) 140, 372 (1982); Phys. Rev. Lett 8, 975 (1982).

[3] S. Carroll, G. Field and R. Jackiw, Phys. Rev. D 411231 (1990).

[4] R. Jackiw and S.-Y. Pi, Phys. Rev. D 68104012 (2003).

[5] A. Lue, L. Wang and M. Kamionkowski, Phys. Rev. Lett. 83, 1506 (1999).

[6] A. N. Redlich, Phys. Rev. D 29, 2366 (1984)

[7] M. Gomes, V. O. Rivelles and A. J. Silva, Phys. Rev. D 41, 1363 (1990)

[8] M. Gomes, R. S. Mendes, R. F. Ribeiro and A. J. Silva, Phys. Rev. D 43, 3516 (1991)

[9] T. Mariz, J. R. S. Nascimento, R. F. Ribeiro and F. A. Brito, Phys, Rev. D 68, 087701, (2003)

[10] L. Alvarez-Gaumé, S. Della Pietra and G. Moore, Ann. Phys. (NY) 163, 288 (1985).

[11] I. Vuorio, Phys. Lett B 175176 (1986).

[12] D. Colladay and V. A. Kostelecký, Phys. Rev. D 58, 116002 (1998).

[13] S. Coleman and S.L. Glashow, Phys. Rev. D 59, 116008 (1999).

[14] R. Jackiw and V.A. Kostelecký, Phys. Rev. Lett. 82, 3572 (1999).

[15] J.M. Chung and P. Oh, Phys. Rev. D 60, 067702 (1999).

[16] J.M. Chung, Phys. Rev. D 60, 127901 (1999). 
[17] W.F. Chen, Phys. Rev. D 60, 085007 (1999).

[18] M. Pérez-Vitoria, Phys. Rev. Lett. 83, 2518 (1999).

[19] J.M. Chung, Phys. Lett. B 461, 318 (1999).

[20] G. Bonneau, Nucl. Phys. B 593, 398 (2001).

[21] Yu. A. Sitenko, Phys. Lett. B 515, 414 (2001).

[22] M. Pérez-Victoria, J. High Energy Phys. 04, 032 (2001).

[23] M. Chaichian, W.F. Chen, and R. González Felipe, Phys. Lett. B 503, 215 (2001).

[24] J.M. Chung and B.K. Chung, Phys. Rev. D 63, 105015 (2001).

[25] A.A. Andrianov, P. Giacconi, and R. Soldati, J. High Energy Phys. 02, 030 (2002).

[26] D. Bazeia, T. Mariz, J. R. Nascimento, E. Passos and R. F. Ribeiro, J. Phys. A 364937 (2003)

[27] G. t'Hooft and M.J.G. Veltman, Nucl. Phys. B 44, 189 (1972).

[28] L. Alvarez-Gaumé and E. Witten, Nucl. Phys. B234, 269 (1984). 